\title{
Bamboo nodes associated with mixed connective tissue disease as a cause of hoarseness
}

\author{
Cornelia Schwemmle • Hans-Heinrich Kreipe • \\ Torsten Witte $\cdot$ Martin Ptok
}

Received: 3 July 2011/ Accepted: 22 October 2011/Published online: 16 November 2011

(C) The Author(s) 2011. This article is published with open access at Springerlink.com

\begin{abstract}
Vocal fold lesions related to autoimmune diseases are rheumatoid nodules and, to a lesser extent, bamboo nodes. Mostly transverse, they are located in the middle third of the vocal cord and exhibit a yellowish appearance. The characteristic shape of these lesions led to their name. These vocal fold deposits may interfere with the normal vibratory cycle during phonation and thus may be an unusual cause of hoarseness. We present a 43-yearold woman with known mixed connective tissue disease and a dysphonia. Laryngostroboscopy showed bamboo nodes as described above. We applied several laryngeal injections of cortisone as described previously in the literature. Since this treatment did not lead to a sufficient voice improvement, we attempted to surgically remove the deposits. After the surgery, the voice improved considerably. In all patients with rheumatic diseases who suffer from a rough, breathy, or unstable voice, a laryngostroboscopic examination should be done. If, however, a bamboo node lesion of the vocal folds is found by the laryngologists, an associated autoimmune disorder must be assumed, and adequate diagnostic procedures have to be initiated. Local laryngeal injections (1-3 times) with steroids should be the first line of therapy. In unsuccessful cases,
\end{abstract}

C. Schwemmle $(\varangle) \cdot$ M. Ptok

Department of Pedaudiology and Phoniatrics,

Medical University Hannover, Carl-Neuberg-Str. 1,

30625 Hannover, Germany

e-mail: schwemmle.cornelia@mh-hannover.de

H.-H. Kreipe

Department of Pathology, Medical University Hannover,

Hannover, Germany

T. Witte

Department of Immunology and Rheumatology,

Medical University Hannover, Hannover, Germany subsequent surgery can be a useful treatment of bamboo nodes to stabilize and improve voice quality.

Keywords Dysphonia - Bamboo nodes - Treatment . Mixed connective tissue disorder

\section{Introduction}

So far, there are relatively few published cases of rheumatoid nodule formation involving vocal folds or other parts of the larynx. Raven et al. [1] were the first to describe small submucous rheumatoid nodules in the larynx and epiglottis. In 1993, Hosako described a case of systemic lupus erythematosus (SLE) in which the submucosal lesions in the vocal folds did not resemble the rounded nodules, and he described these unusual lesions as "bamboo-joint-like" [2]. Murano used the term "vocal fold bamboo nodes" to simplify the nomenclature of the same feature [3].

Here we present symptoms, clinical findings, and histopathology in a patient with bamboo nodes, which are associated with mixed connective tissue disease (MCTD). Therapeutic procedures of bamboo nodes are discussed.

\section{Materials and results}

Case report

In June 2004, a 43-year-old female primary school teacher had a sudden onset of hoarseness. She consulted an otolaryngologist and received antibiotics, which did not improve her voice quality. In November 2004, she was first examined in our Department of Pedaudiology and 
Phoniatrics at the Medical School Hannover. A mixed connective tissue disease (MCTD) had been diagnosed in 1998. Raynaud's phenomenon has been present until today. The blood examination had revealed antinuclear (ANA) and ribonuclease-sensitive (RNP) antibodies. Recent X-ray of the thorax showed subpleural emphysematous signs in the upper lung and basal interstitial reticular changing. As recommended by her immunologist, she did not receive prednisolone as a maintenance dose. The most prominent perceptual feature was an unstable voice with intermittent roughness. She also complained that an increased effort was required to produce voice.

The rigid laryngeal endoscopy revealed bilateral whitish transverse band stripes in the submucosa, which lent a slightly protruded aspect to the vocal folds. One lesion was found at the junction of the middle and posterior thirds of the right vocal fold, and another one was observed in the middle of the left vocal fold (Fig. 1). The site of the bilateral lesion became more evident during the stroboscopic examination. Moreover, the stroboscopic view during chest voice phonation showed a decreased amplitude vibration throughout the vocal folds except for the lesion, where no vibratory pattern between the portions anterior and posterior to the transverse lesion in the vocal fold was visible in the continuous image of the videostroboscopy.

Maximum phonation time was $12.9 \mathrm{~s}$ (mean $21 \mathrm{~s}$, normal $\geq 7 \mathrm{~s}$ ). Real-time analysis of connected speech during reading a short text ("Der Nordwind und die Sonne"; a German equivalent to the Rainbow passage) showed a mean fundamental frequency of $167 \mathrm{~Hz}$ (adult women range 190-240 Hz) and an interperiodic irregularity [4] of $26.57 \%$ (normal $<11 \%$ ).

On the basis of other case reports in the literature, we suggested an intralesional steroid injection. This was

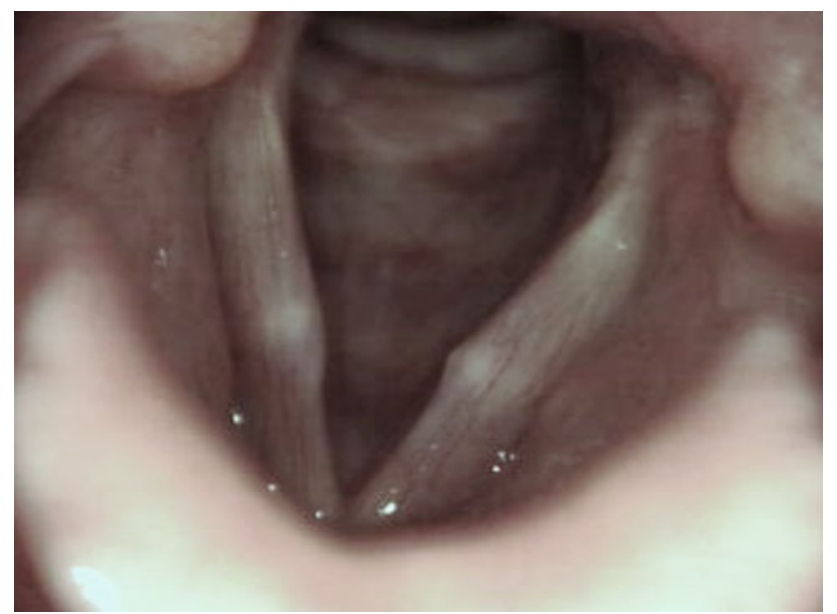

Fig. 1 Endoscopic view of larynx before surgical therapy: bilateral whitish transverse band stripes on both sides of vocal folds applied four times using prednisolone $(5 \mathrm{mg}$ ) in both vocal folds. Inter-injection interval was 1 week. After the second injection, the voice improved. Since the patient still wished further improvement, we injected steroids for the third time, but unfortunately the voice became hoarse again. We decided to apply a fourth injection, but the voice quality did not change after that.

Finally, we decided to perform an excision of the lesions. Microlaryngoscopic surgery was done under general anesthesia in January 2005. The surgical technique consisted of an incision lateral to the lesion on the superior surface of the vocal fold, followed by exploration of the mucosal layer along and under the lesion. A hard and crumbly whitish substance without an encapsulating structure was found under the mucosal layer, which joint the vocal ligament transversally. Because of the infiltration depth, the substance could not be removed completely on both sides without endangering the $\mathrm{m}$. vocalis.

The histopathologic examination of vocal cord biopsies (hematoxylin-eosin staining) revealed an extensive fibrinoid necrosis surrounded by a rim of histiocytes in a palisading fashion. The histiocyte-rich granulomatous infiltrate with a necrotic core closely resembled rheumatoid nodules. On morphologic grounds, a connective tissue disease was suspected in the histopathology report (Fig. 2).

\section{Follow-up}

In March 2005, 2 months after surgery, the patient's voice was better than before surgery and acceptable for normal and professional voice use. She returned to her teaching activities. Following surgery, the patient received speech training for several months (20 times) in order to stabilize

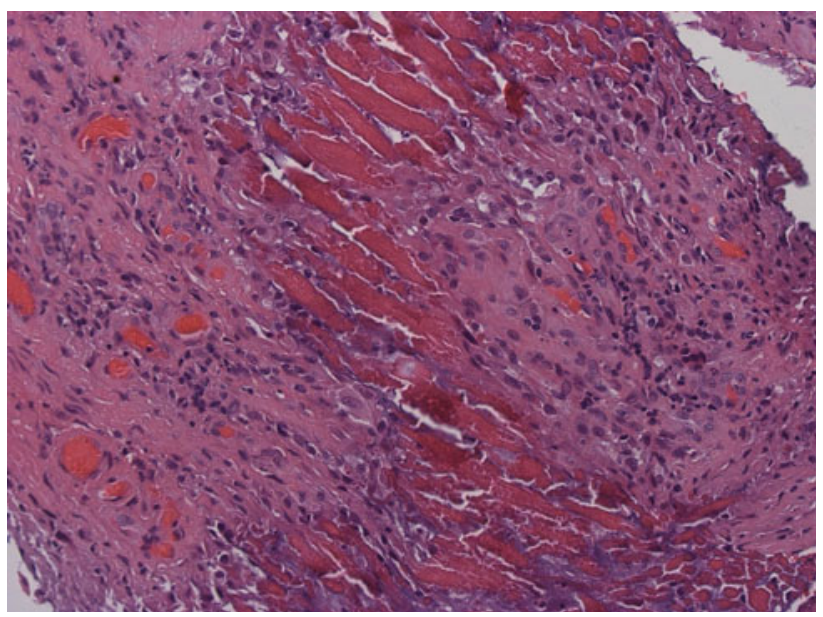

Fig. 2 Pathology of vocal fold lesion: fibrinoid necrosis surrounded by a rim of histiocytes in a palisading fashion. Histiocyte-rich granulomatous infiltrate with a necrotic core close. (Hematoxylin and eosin stain $\times 20$ ) 
voice quality. One month after surgery, a laryngoscopic examination showed reduced bilateral whitish transverse band stripes in the submucosa (Fig. 3). The stroboscopic view during chest voice phonation demonstrated the already-known decreased amplitude vibration throughout the vocal folds with the exception of the lesion on the left side. On the right side, a small vibratory pattern between the portions anterior and posterior to the transverse lesion in the vocal fold was visible again in the continuous image of the videostroboscopy. Maximum phonation time was $14.5 \mathrm{~s}$. Real-time analysis of speech showed a mean fundamental frequency of $195 \mathrm{~Hz}$ and an interperiodic irregularity [4] of $10.9 \%$ (normal $<10 \%$ ). In comparison with data from before the first conservative treatment, there is an improvement in all aspects of voice quality as measured by objective means.

In August 2010, the stroboscopic view during chest voice phonation demonstrated the already-known decreased amplitude vibration throughout the vocal folds with the exception of the lesion on the left side. On the right side, a small vibratory pattern between the portions anterior and posterior to the transverse lesion in the vocal fold was visible again in the continuous image of the videostroboscopy. Maximum phonation time was $15.0 \mathrm{~s}$. Real-time analysis of speech showed a mean fundamental frequency of $205 \mathrm{~Hz}$ and an interperiodic irregularity [4] of $11.84 \%$ (normal $<11 \%$ ). With an average follow-up period of 6 years, we noted a long-term stabilization of her voice quality. Short intervals of speech therapy for a year now have been helping to keep good laryngeal function. At the moment, she is working full-time as a teacher.

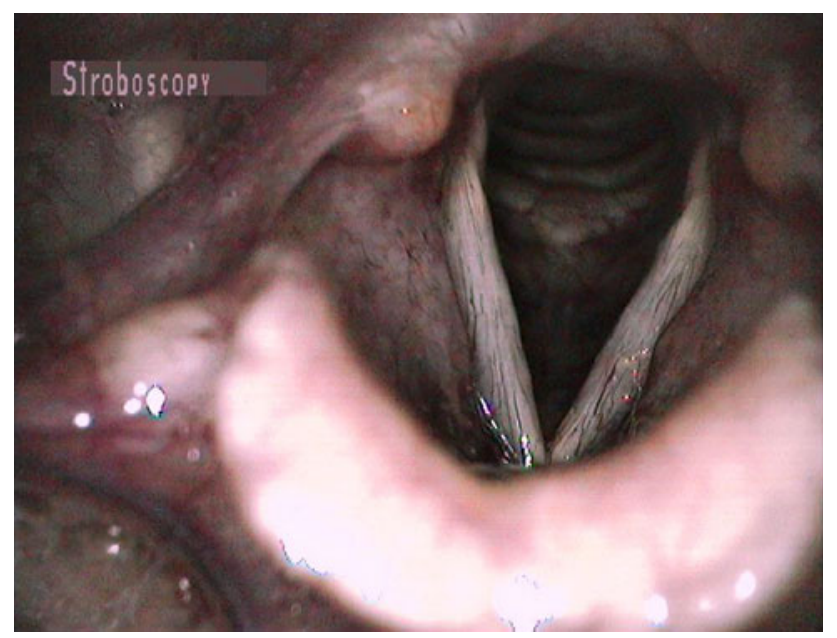

Fig. 3 Endoscopic view of larynx after surgical therapy: bilateral reduced whitish transverse band stripes on both sides of vocal folds

\section{Discussion}

Laryngeal manifestations in autoimmune diseases have been known for a long time and may include cricoarytenoid arthritis, mucous inflammation, subglottic stenosis, vasculitis, laryngeal edema, vocal fold paralysis, infection, or vocal fold lesions such as vocal fold rheumatoid nodules [5]. Especially bamboo nodes are poorly recognized as a laryngeal manifestation of autoimmune disease. Rheumatoid nodules are most likely to appear in areas subject to repeated micro trauma. It is at the midpoint of the membranous vocal folds that the contact forces are greatest during vibration [6]. This possibly explains why rheumatic deposits develop at this specific site and not elsewhere [7].

In many cases of bamboo nodes, a systemic lupus erythematous (SLE) [7-9] was diagnosed, but these lesions have also been described in patients with rheumatoid arthritis, Sjögren's syndrome, Hashimoto's thyroiditis, and progressive systemic sclerosis [7]. Occasionally, bamboo nodes appear in advance of other symptoms $[2,10]$ with serologic abnormalities as the only associated findings. These may include elevations in the sedimentation rate, rheumatoid factor, Sjögren's antibodies, and antinuclear antibody titers, as well as hypergammaglobulinemia. Progression to multisystemic connective tissue disease after the diagnosis of bamboo nodes is not inevitable, and in some patients, bamboo nodes appear to remain the only sign of disease for years [11]. Follow-up over several years also suggests that bamboo nodes are related to autoimmune activity [3, 12].

In literature review, all patients were women, and in most of the cases, an autoimmune disease had been diagnosed before the presence of bamboo nodes. Although the number of the cases described in the literature is still scarce, bamboo nodes are most frequently described in SLE patients (Table 1).

Different strategies of therapies are discussed in the literature

\section{Conservative}

Medical therapy: Murano believes that bamboo nodes are a sign of activity of the autoimmune disorder [3]. Thus, he and others believe that clinical treatment with (systemic) steroids should be tried first $[3,8,11]$ Murano also recommended voice rest. Hosako-Naito described a postoperative treatment with oral steroids, which helped to reduce recurrence [8]. In general, the aim of conservative therapy of rheumatoid nodules with local injection of prednisolone in the lesions is a reduction of the lesion masses and the inflammatory process. In addition to previous reports, we 
Table 1 Review of the literature on bamboo nodes

\begin{tabular}{|c|c|c|c|c|}
\hline \multirow{2}{*}{$\frac{\text { No. }}{1}$} & \multicolumn{2}{|c|}{ Sex/age } & \multirow{2}{*}{$\begin{array}{l}\text { Disease } \\
\text { SLE }\end{array}$} & \multirow{2}{*}{$\frac{\text { Treatment }}{\text { Steroid drugs }}$} \\
\hline & $\mathrm{F} 28$ & Hosako [2] & & \\
\hline 1 & F51 & Nishinarita et al. [14] & UCTS, autoimmune hepatitis & Steroid drugs \\
\hline 1 & $\mathrm{~F} / 51$ & Tsunoda et al. [10] & SLE, autoimmune hepatitis & Steroid drugs \\
\hline \multirow[t]{4}{*}{4} & $\mathrm{~F} / 28$ & Hosako-Naito et al. [8] & SLE & Surgery \\
\hline & $\mathrm{F} / 48$ & & Hashimoto & Surgery \\
\hline & $\mathrm{F} / 32$ & & Progressive systemic sclerosis & Surgery \\
\hline & $\mathrm{F} / 27$ & & High ANA & Surgery \\
\hline \multirow[t]{2}{*}{2} & $\mathrm{~F} / 28$ & Murano et al. [3] & SLE & Surgery \\
\hline & $\mathrm{F} / 36$ & & Sjögren's disease & Surgery and steroid drugs \\
\hline \multirow[t]{5}{*}{19} & $\mathrm{~F} / ?$ & Perouse et al. [13] & 2x RA & \\
\hline & & & $3 x$ SLE & \\
\hline & & & 2x Sjögren's disease & \\
\hline & & & MCTD & \\
\hline & & & 11 no autoimmune disease & All patients surgery \\
\hline \multirow[t]{7}{*}{11} & All F & Ylitalo et al. [7] & $5 x$ RA & \\
\hline & Age 16 & an 37 years & SLE & \\
\hline & & & Progressive systemic sclerosis & \\
\hline & & & 2x Sjögren's disease & \\
\hline & & & MCTD & \\
\hline & & & Arthralgia, myalgia & Medical treatment unknown \\
\hline & & & Proteinuria & 5 of all patients surgery \\
\hline \multirow[t]{5}{*}{4} & $\mathrm{~F} / 36$ & Ramos et al. [15] & MCTD & Steroid drugs \\
\hline & $\mathrm{F} / 31$ & & MCTD & Steroid drugs \\
\hline & $\mathrm{F} / 46$ & & SLE & Steroid drugs \\
\hline & $\mathrm{F} / 35$ & & SLE & Steroid drugs \\
\hline & $\mathrm{F} / 28$ & & SLE & Steroid drugs \\
\hline 1 & $\mathrm{~F} / 24$ & Immerman et al. [11] & RA & Steroid drugs \\
\hline 1 & $\mathrm{~F} / 46$ & Schwemmle et al. [12] & MCTD & Steroid drugs (lar. injection) \\
\hline \multirow[t]{2}{*}{2} & $\mathrm{~F} / 29$ & Hilgert et al. [5] & MCTD & Speech therapy \\
\hline & $\mathrm{F} / 31$ & & MCTD & Speech therapy and steroid drugs \\
\hline 1 & $\mathrm{~F} / 38$ & Li et al. [16] & Elevated ANA, no clinical signs & Surgery \\
\hline
\end{tabular}

$S L E$ systemic lupus erythematosus, UCTS undifferentiated connective tissue syndrome, $R A$ rheumatoid arthritis, MCTD mixed connective tissue syndrome, lar Laryngeal

believe that local corticoid injection into the vocal folds does have a precise effect and should be tried first [12].

Speech therapy: In contrast to other reports, Hilgert proposed a speech therapy first without additional laryngeal injection steroid therapy [5]. Perouse described the possibility of a speech therapy after laryngeal surgery to retrain normal laryngeal function [13]. We recognized improved voice, endoscopic results, and voice range profiles after surgery. Speech therapy after surgery may have been stabilizing voice quality until today.

\section{Surgery}

Murano recommended careful surgery without harming the vocal ligament, if there is an incomplete disappearance of the lesion or if the patient has an urgent reason to improve voice quality, and if the underlying disease is well controlled [3]. In contrast, Hosako-Naito discussed a complete excision of the lesion to reduce a recurrence [8]. Perouse reported 19 cases treated by surgery with a favorable outcome [13]. The recommended extent of surgery was not described. In general, the aim of surgery in these patients is to diminish instability and to recreate a better vibratory pattern. In our case, careful surgery with reduction of the lesion on both sides was done. After this treatment, the voice's stability and clearness improved so much that the patient could carry on her teaching activities.

Nevertheless, it is not yet clear which treatment leads to the best outcome. This requires further investigation. 


\section{Conclusion}

Rheumatologists and otolaryngologists both play an important role in the care and the clinical investigation of patients with autoimmune diseases and hoarseness, as the disorder may manifest itself with hoarseness before the diagnosis of an autoimmune disease can be established. Although the pathophysiology is not yet clear, highly demanding voice use may be related to the appearance of bamboo nodes in autoimmune disease patients. This case report may add some leads how to treat patients with bamboo nodes:

1. Laryngeally injected steroids should be tried in the first place. They seem to be effective in achieving a good outcome, resulting in voice improvement and alleviation of clinical symptoms, but they should not be applied more than three times, with a 14-day interval between injections.

2. As the second line, after ineffective laryngeal injection of steroids, surgery with careful partial excision and reduction of the lesions is recommended.

3. A speech therapy as an adjunct should be considered to further stabilize voice quality after laryngeal steroid injection/surgery or as a compensation procedure in cases that cannot be treated sufficiently with either steroid injections or surgery.

\section{Conflict of interest None.}

Open Access This article is distributed under the terms of the Creative Commons Attribution Noncommercial License which permits any noncommercial use, distribution, and reproduction in any medium, provided the original author(s) and source are credited.

\section{References}

1. Raven RW, Patkes WF, Woodhouse PL (1948) The necrobiotic nodules of rheumatoid arthritis. Ann Rheum Dis 7:63-75
2. Hosako Y, Nakamura M, Tayama N, Mizuno M, Matsunaga A, Niimi S, Hirose H, Hagino S (1993) Laryngeal involvements in systemic lupus erythematosus. Larynx Jpn 6:171-175

3. Murano E, Hosako-Naito Y, Tayama N, Oka T, Miyaji M, Kumada M, Niimi S (2001) Bamboo node: primary vocal fold lesion as evidence of autoimmune disease. J Voice 15:441-450

4. Fourcin A, Abberton E, Miller D, Howells D (1995) Laryngograph: speech pattern element tools for therapy, training and assessment. Eur J Disord Commun 30:101-115

5. Hilgert E, Toleti B, Kruger K, Nejedlo I (2008) Hoarseness due to bamboo nodes in patients with autoimmune diseases: a review from the literature. J Voice 22:343-350

6. Titze IR (1994) Mechanical stress in phonation. J Voice 8:99-105

7. Ylitalo R, Heimbürger M, Lindestad PA (2003) Vocal fold deposits in autoimmune disease-an unusual cause of hoarseness. Clin Otolaryngol 28:446-450

8. Hosako-Naito Y, Tayama N, Niimi S, Aotsuka S, Miyaji M, Oka T, Fujinami M, Kitahara N (1999) Diagnosis and physiopathology of laryngeal deposits in autoimmune disease. J Otorhinolaryngol Relat Spec 61:151-157

9. Speyer R, Speyer I, Heijnen M (2008) Prevalence and relative risk of dysphonia in rheumatoid arthritis. J Voice 22:232-237

10. Tsunoda K, Soda Y (1996) Hoarseness as the initial manifestation of systemic lupus erythematosus. J Laryngol Otol 110:478-479

11. Immerman S, Sulica L (2007) Bamboo nodes. Otolaryngol Head Neck Surg 137:162-163

12. Schwemmle C, Ptok M (2007) Bamboo nodes as the cause of dysphonias in autoimmune diseases. HNO 55:564-568

13. Perouse R, Coulombeau B, Cornut G, Bouchayer M (2001) "Bamboo nodes": a clinical study of 19 cases. Rev Laryngol Otol Rhinol 122:299-302

14. Nishinarita M, Ohta S, Uesato M, Oka Y, Kamoshita T, Takahashi A (1995) Undifferentiated connective tissue syndromes (UCTS) accompanied by laryngeal involvement and autoimmune hepatitis. Nihon Rinsho Meneki Gakkai Kaishi 18:559-565

15. Ramos HV, Pillon J, Kosugi EM, Fujita R, Pontes P (2005) Laryngeal assessment in rheumatic disease patients. Braz J Otorhinolaryngol 71:499-503

16. Li L, Saigusa H, Nakazawa Y, Nakamura T, Komachi T, Yamaguchi S, Liu A, Sugisaki Y, Shinya E, Shen H (2009) A pathological study of bamboo nodule of the vocal fold. J Voice 24 : 738-741 